\title{
THE EXTRAGALACTIC BACKGROUND INCLUDED IN IRAS POINT SOURCE SAMPLES
}

\author{
E. J. A. Meurs \\ ESO \\ Karl-Schwarzschild-Str. 2 \\ D-8046 Garching bei München \\ $F R G$
}

R. T. Harmon
Astronomy Dept.
105-24 Caltech
Pasadena, CA 91125, USA

\author{
H.-M. Adorf \\ $S T-E C F / E S O$ \\ Karl-Schwarzschild-Str. 2 \\ D-8046 Garching bei München \\ FRG
}

\begin{abstract}
The sky survey performed by the IRAS satellite provides a very homogeneous and almost complete view of the sky at infrared wavelengths. Suitable methods from multivariate statistics and decision theory are employed to extract the extragalactic background included in the IRAS Point Source Catalog. The resulting map comprehensively represents the distribution of extragalactic objects on the sky. The various selections may be used to assess large-scale structure in the distribution of galaxies and the total infrared background due to galaxies, to follow such structures closer to the Galactic Plane than is possible with optical data and to address cosmological questions (e.g., Harmon, Lahav, and Mauers 1987). These investigations are to be extended to the more sensitive Co-Added Catalog.
\end{abstract}

\section{INTRODUCTION}

A sample of galaxies that has a high degree of homogeneity as well as large sky coverage is required for various studies dealing with the distribution of galaxies at large, including estimates of their contribution to the background light at particular wavelengths. The sky survey performed by the IRAS satellite at infrared wavelengths provides a database with such properties. Therefore it is of interest to isolate the extragalactic sources contained in the IRAS Point Source Catalog (PSC), for which purpose a suitable selection method has to be developed.

\section{SOURCE SELECTION}

Various studies of the contents of the PSC have shown that, in general, stars are bright at 12 and $25 \mu \mathrm{m}$ while galaxies are bright at 60 and $100 \mu \mathrm{m}$. Therefore we selected from Version II of the PSC the sources with high or moderate quality fluxes at 60 and $100 \mu \mathrm{m}$. The resulting distribution of data points can be broken up into a few categories of sources: stars, "cirrus," "thin plane," and extragalactic sources (for details see Meurs and Harmon 1988).

As described in Adorf and Meurs (1988) and Meurs, Adorf, and Harmon (1988), a Bayesian classifier can be constructed for the source categories involved from appropriate training sets, obtained on the basis of the source distribution in galactic coordinates. The source characteristics that are included in this process are the fluxes in the four IRAS bands as represented by three flux ratios ("colors": 12/25, 25/60,60/100) and one flux (at $100 \mu \mathrm{m}$ ). The colors are defined as $C_{m n}=\log \left(F_{m} / F_{n}\right)$, where $F_{1}$ is the flux in band $1(12 \mu \mathrm{m})$, etc.

The extragalactic objects may be characterized by the center coordinate $\left(C_{12}, C_{23}, C_{34}, \log F_{4}\right)=(-0.0507,-0.492,-0.337,0.349)$ and the variance-covariance matrix of the (Gaussian) density function fitted to the distribution of data points in feature space. 


\section{EXTRAGALACTIC BACKGROUND}

The extragalactic background that is contained in the IRAS PSC has thus been extracted and the resulting map (Figure 1) represents comprehensively the distribution of extragalactic objects on the sky. Features in the distribution also visible on maps constructed from optical data (see Meurs and Harmon 1988) include galaxy chains and clusters and the Supergalactic Plane.

The zone of avoidance is in the IRAS data restricted to an elliptically shaped region with semi-axes of $-90^{\circ}$ and $\sim 12^{\circ}$-much smaller than in optical data where the zone is a band extending all over the sky. For the IRAS zone of avoidance, source confusion is likely to be a factor of greater importance than extinction. Figure 1 demonstrates the large degree of homogeneity of the IRAS data whereas optically the boundaries between different catalogues are discernible. The impression of homogeneity is further assisted by the fact that the IRAS PSC data refer almost exclusively to spirals, which are less clustered than ellipticals, and that the infrared luminosity function of galaxies is broader than the optical one.

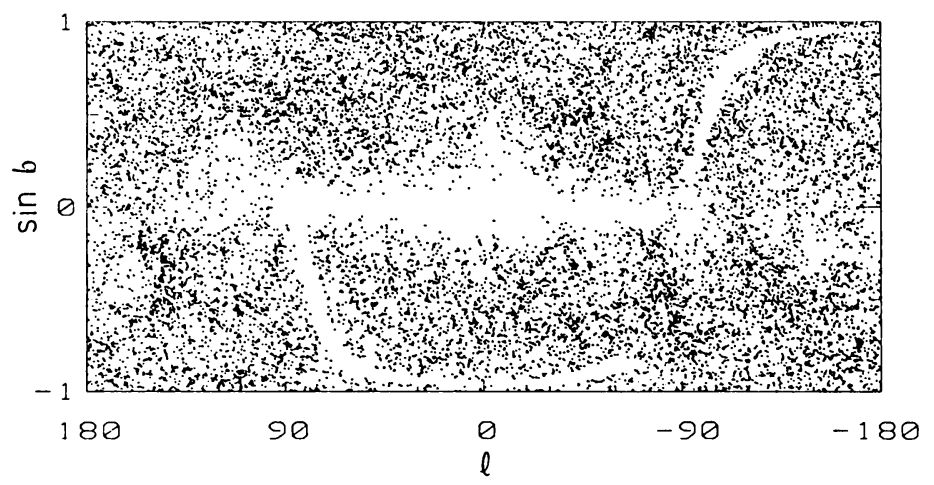

Figure 1. Large-scale distribution of 'galaxies' as selected by the maximum-a-posteriori classifier out of the 33435 pre-selected objects.

The success rate of a selection of extragalactic objects as presented in this paper may be estimated in several ways, as discussed in Meurs and Harmon (1988). They inferred that some $90 \%$ of the selected sources actually are galaxies while some $10 \%$ of all extragalactic PSC sources escape selection. Only within about $5^{\circ}$ from the galactic plane and in some regions in the Gould Belt the contamination by cirrus sources may increase to some $40 \%$.

\section{REFERENCES}

Adorf, H.-M., and Meurs, E.J.A. 1988, in Large-Scale Structures in the Universe - Observational and Analytical Methods, ed. W.C. Seitter, H.W. Duerbeck, and M. Tacke (Heidelberg, FRG: Springer), p. 315.

Harmon, R.T., Lahav, O., and Meurs, E.J.A. 1987, Mon. Not. Royal Astron. Soc. 228, 5P.

Meurs, E.J.A., and Harmon, R.T. 1988, Astron. Astrophys. 206, 53.

Meurs, E.J.A., Adorf, H.-M., and Harmon, R.T. 1988, in Astronomy from Large Databases: Scientific Objectives and Methodological Approaches, ed. F. Murtagh and A. Heck (Garching, FRG: ESO), p. 49. 